\title{
Evaluation of Multi-Asset Investment Strategies with Digital Assets
}

\author{
Bachelor's Thesis submitted \\ to \\ Prof. Dr. Wolfgang Karl Härdle \\ Prof. Dr. Weining Wang \\ Advisor: Dr. Alla Petukhina \\ Humboldt-Universität zu Berlin \\ School of Business and Economics \\ IRTG 1792 High Dimensional Nonstationary Time Series \\ by
}

\section{Erin Dirk Sprünken}

(581608)

in partial fulfillment of the requirements

for the degree of

Bachelor of Science (B.Sc.) in Economics

Berlin, September 8, 2019
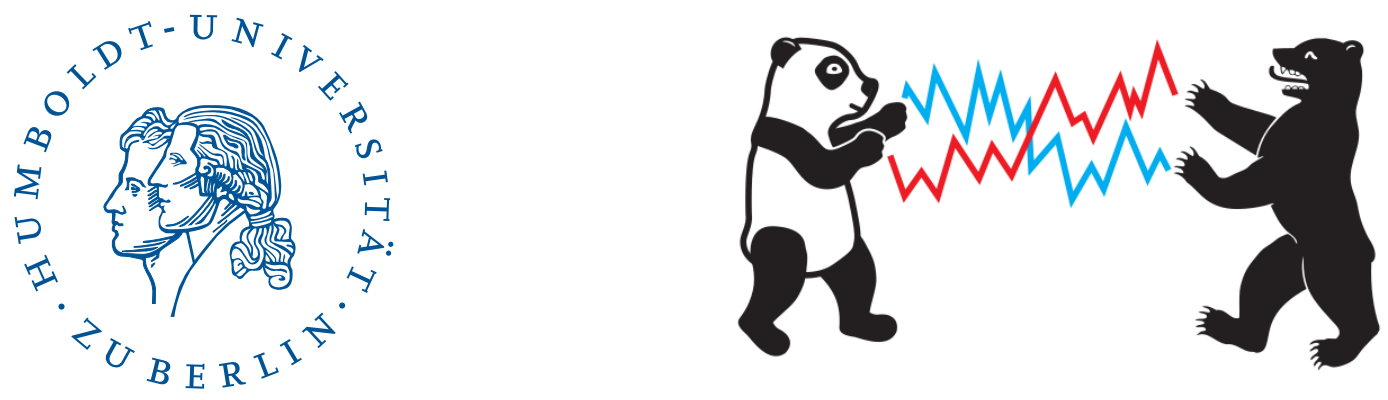
I want to thank my family and friends for their continuous encouragement during my studies.

Furthermore, I want to thank Prof. Dr. Wolfgang Karl Härdle and Dr. Alla Petukhina for their advisory, support and feedback. 


\section{Contents}

\begin{tabular}{llr}
\hline 1 & Introduction & 1
\end{tabular}

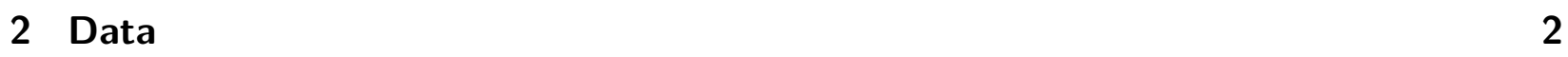

$\begin{array}{llr}3 & \text { Methodology } & 4\end{array}$

3.1 Parameter-Estimation . . . . . . . . . . . . . . . . . 5

3.1 .1 Arithmetic Mean (AM) . . . . . . . . . . . . . . . . . . . 5

3.1 .2 Geometric Mean (GM) . . . . . . . . . . . . . . . . . . . . . . 5

3.1 .3 Variance-Covariance-Matrix (AM/GM) . . . . . . . . . . . . . . . . 6

3.1 .4 Bayes-Stein Shrinkage Estimator (BS) . . . . . . . . . . . . . . . . . . 6

3.2 Portfolio Optimization . . . . . . . . . . . . . . . . . . . . . . . . . . . . . . . . 7

$3.2 .1 \quad$ Equally Weighted (Naive) . . . . . . . . . . . . . . . . . . . . . . . . . . 7

3.2.2 Mean-Variance (Modern Portfolio Theory - MPT) . . . . . . . . . . . . 8

3.2.2.1 Target-Return . . . . . . . . . . . . . . . . . 8

3.2.2.2 Sharpe-Ratio-Maximization (Tangency Portfolio) . . . . . . . . 8

3.2.2.3 Certainty Equivalent-Maximization . . . . . . . . . . . . . . . 9

3.2.2.4 Global Minimum Variance . . . . . . . . . . . . . . . . . . . . 10

3.2 .3 Geometric Mean Maximization . . . . . . . . . . . . . . . . . . . . . . 10

3.2.4 Conditional Value-at-Risk Minimization (CVaR) . . . . . . . . . . . . . . 11

3.2.5 LIBRO . . . . . . . . . . . . . . . . . . . . . . . . . . . . . . . . . . . 12

3.3 Performance Metrics . . . . . . . . . . . . . . . . . . . . . . . . . . . . . . . . 13

3.3 .1 Certainty Equivalent . . . . . . . . . . . . . . . . . . . . . . . . 13

3.3 .2 (Adjusted) Sharpe Ratio . . . . . . . . . . . . . . . . . . . . . . . . . . 13

3.3 .3 Turnover . . . . . . . . . . . . . . . . . . . . . . . . . . . 14

3.3 .4 Terminal Return . . . . . . . . . . . . . . . . . . . . . . . . . . 14

3.4 Significance . . . . . . . . . . . . . . . . . . . . . . . . . . . . . . 14

$\begin{array}{lll}4 & \text { Empirical Analysis } & 15\end{array}$

$\begin{array}{llr}5 & \text { Conclusion } & 27\end{array}$

$\begin{array}{lr}\text { References } & 29\end{array}$

Appendix A - Asset Classes 32

Appendix B - Descriptive Statistics 33 


\section{List of Tables}

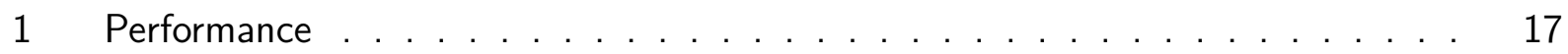

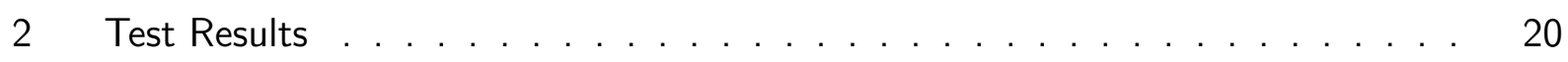

3 Assets . . . . . . . . . . . . . . . . . . . . . . . . . . . . . . . . 32

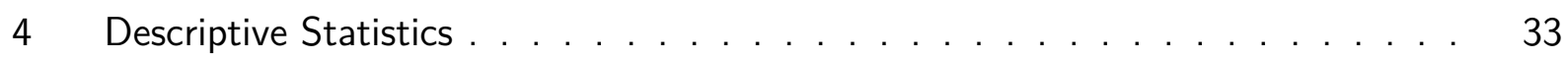

5 Names . . . . . . . . . . . . . . . . . . . . . . . . 34 


\section{List of Figures}

1 Cumulative Performances . . . . . . . . . . . . . . . . . . . . . . . . 22

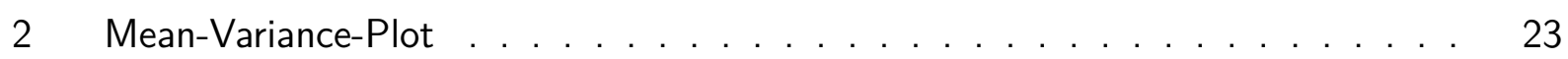

$3 \quad$ Boxplots (Mean and Standard Deviation) . . . . . . . . . . . . . . . . . . 24

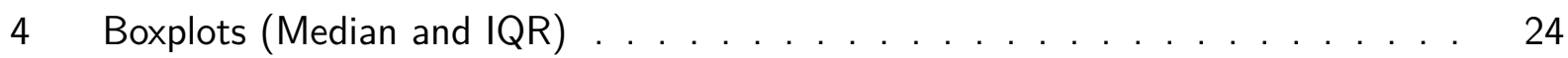

5 Boxplots (Geometric Mean and Terminal Return) . . . . . . . . . . . . . . 25

$6 \quad$ Density . . . . . . . . . . . . . . . . . . . . . . . . . . . 26

$7 \quad$ Tangency Portfolio (AM) $\ldots \ldots \ldots \ldots$ 


\section{Introduction}

For a very long time the optimal allocation of wealth has been an important topic for mankind and is still developing. DeMiguel et al. (2009) cite a rabbiner from the fourth century who already proposed a rule on how to split wealth across assets. Since then, many researchers have tried to find the best portfolio strategy. A remarkable step was taken when the Modern Portfolio Theory ("MPT") arised in 1952 with Markowitz' Mean-Variance analysis for which he was granted the Nobel Memorial Prize in Economic Sciences, see Markowitz (1952). 50 years later Fabozzi et al. (2002) conclude that the MPTs importance will not vanish and that it will have permanent presence in financial research and practice. However, they also note that the MPT is a normative theory, which means that it says how optimal investing should be done under perfect information. As DeMiguel et al. (2009) note, the strategy suffers from poor out of sample performance. Reasons for this are presumably that MPT tends to estimate extreme weights and is very sensitive to changes in its inputs. Furthermore, the estimation of these inputs may be another fallacy. This has been the reason for continuous development of portfolio optimization, as for example in Jorion (1986), Clarke et al. (2013), Bessler et al. (2017), DeMiguel et al. (2009), Estrada (2010), Trimborn et al. (2019) and Petukhina et al. (2018).

This paper is comparing and evaluating different optimization techniques under several aspects such as multiple asset classes, different markets and different estimators as well as a specific comparative view on the naive (equally weighted) portfolio.

One important aspect is that this thesis uses different asset classes in the empirical section. The general motivation of diversifying portfolios is to exploit correlation structures among assets, markets or asset classes. This can be helpful in increasing the return or decreasing the risk of the portfolio, see Elton et al. (2003). Due to the rise of alternative investments such as cryptocurrencies this paper will include cryptocurrencies as a new asset class. As Glaser et al. (2014) note there is still a discussion whether these should be treated as alternative assets or currencies. Although this discussion is still ongoing, there exists recent research dealing with cryptocurrencies as assets, see Trimborn et al. (2019) and Petukhina et al. (2018). Both include these in portfolios together with traditional assets such as stocks, bonds and commodities. However, Klein et al. (2018) studied the properties of Bitcoin as an asset and specifically compared it to gold. They found that, though there is a similarity to major precious metals in its response to market shocks, Bitcoin as an asset differs from other conventional assets. This backs the statement that Bitcoin and presumably other cryptocurrencies should be treated as alternative assets. 
Commodities were included as alternative assets as well. Typically, portfolios are diversified among asset classes and markets. That is not new, as especially gold is considered as one of the most traditional alternatives to stocks and bonds in portfolios. It is generally known as a "safe haven", meaning that investors tend to buy gold if they fear bear markets or crashes, see Baur and McDermott (2010). Furthermore, this thesis includes palladium, silver, corn, wheat and diamonds as commodities. Whereas palladium and silver are generally categorized as precious metals and also as options for portfolio diversification, this is not the case for diamonds. They are considered as an alternative investment. One possible reason for this might be that, whereas silver and palladium also are widely used in industry, the main demand for diamonds comes from the wedding industry, see Scott and Yelowitz (2010). Thus, it is interesting to include them in empirical research. The motivation for discussing corn and wheat in a portfolio context is that these have not been in the focus of such studies recently. There is little literature which includes such commodities among other assets. Bessler and Wolff (2015) analyzed the benefits of adding commodities to a stock-bond portfolio and found that industrial and precious metals improved performance whereas agriculture did not. As there is much research with respect to trading and speculation on such commodity markets, see Bosch (2017), this was motivating to add corn and wheat to the data used in this thesis and propose a broad investment horizon in the research of portfolio optimization. Therefore, the empirical section of this paper includes stocks, cryptocurrencies as new assets and commodities as traditional assets and as alternative assets as well.

The contribution of this thesis to current research is that another investment universe is studied in the context of portfolio analysis. Multiple asset classes are included, namely stocks, precious metals, commodities, diamonds and cryptocurrencies. Furthermore, a broader spectrum of strategies is investigated in a comparative matter using several different sucess measurements. In addition, different estimators for input parameters are used.

The thesis is structured in the following way: The second section covers the data used for the empirical analysis. Section three explains the methodology of the investing strategies, section four presents the empirical results and section five gives a brief conclusion.

\section{Data}

The dataset used for the empirical analysis of the strategies includes German stocks, commodities and cryptocurrencies. The stocks were obtained from the German SDAX index in its composition 
as of 08.02.2019 (70 stocks) for a timespan from 01.01.2013 to 31.12.2018 (1565 daily observations). The SDAX is a German stock market index for small-cap stocks. However, the index contains the largest 70 companies which are not large enough to be listed within the DAX (largecap index) or the MDAX (mid-cap index). Though, these companies have to fulfill the criteria of the Prime-Standard regulation of the Frankfurt stock exchange. The stock prices and the trading volume are measured in the native currency (EUR) and the frequency of the observations is daily. The source of the data was Bloomberg.

The dataset of cryptocurrencies contains the ten largest by market capitalization as of 16.02.2019. These were obtained from coingecko.com. The daily observations start at 29.04.2013 up to 31.12.2018. In contrast to the German stocks cryptocurrencies also have observations on weekend days. The prices/exchange rates are measured per Unit of USD (i.e.: A price of 6 BTC means that with 1 USD one could buy 6 BTC). The trading volume is also measured in USD.

Due to availability of the data (e.g.: several cryptocurrencies were issued later than others) the set contains a lot of missing values (NA). To have at least some cryptocurrencies but also a large enough set for calculations, the minimum number of cryptocurrencies with complete observations were set to six in this thesis. The list of the included cryptocurrencies can be found in the appendix.

Thus, after removing weekend days and keeping only complete series, the new set of cryptocurrencies contains six different cryptocurrencies and daily observations from 10.08.2015 to 31.12.2018. The SDAX set has been adjusted to the new timespan and non-complete series were removed as well.

In addition, some commodities were chosen as alternative assets to be included into the portfolio optimization. These are palladium, gold, silver, two types of corn, one type of wheat and three types of diamonds. After adjusting the data to those of the SDAX and cryptocurrencies, the final set of these alternative assets starts at 10.08.2015 as well. The source was Thomson Reuters Eikon, used at 04.03.2019. The unit for the respective prices is USD. Data of their trading volume was not available.

The final data contains 886 daily observations for 60 German stocks, six cryptocurrencies and nine commodities. For the purpose of portfolio optimization, one is not directly interested in the prices but rather indirectly through the returns. Furthermore, returns have the convenient 
property of being "standardized" in the sense that they share the same unit (percentage). This is very useful here as the assets are measured in different units (EUR and USD), but also have completely different magnitudes. Thus, daily returns will be calculated and used in this thesis. This reduces the dataset to 885 daily observations. The data of the trading volume has been adjusted in the way that the first observation of the time-adjusted set has been removed. Hence, the volume data contains 885 observations as well but only for 60 German stocks and six cryptocurrencies.

Outliers in the data were not removed, simply for the reason that these sometimes happen at the markets (as for example various crashes on stock markets over time). These are extreme events and it is desirable that a strategy is robust against such tail events instead of neglecting the data points in empirical research. The data was doublechecked and the outlying values were reported by different, independent sources. Especially the cryptocurrencies contain these extreme values.

The formula to obtain daily returns from prices is the following:

$$
r_{i, t}=\frac{P_{i, t}-P_{i, t-1}}{P_{i, t-1}}=\frac{P_{i, t}}{P_{i, t-1}}-1
$$

The index $i$ denotes asset $i$ of a given set of assets, $t$ is the time index which corresponds to a day and $P$ is the respective price.

\section{Methodology}

In this section the theoretical background and methodology of the thesis is explained. It starts with a brief overview of the notation used, followed by the estimators for the necessary parameters. Afterwards, a description of the strategies takes place. For each strategy the intuition as well as the mathematical defininition is given. Lastly, the performance measurements used to evaluate the allocation techniques are explained.

$T$ represents the number of available observations. This is equivalent to the total number of days in the data.

$N$ is the number of risky assets. $\mu$ is a $N \times 1$ vector of expected returns of these assets and $r$ the true, realized returns.

$\Sigma$ is the $N \times N$ variance-covariance matrix of the same risky assets.

$r_{f}$ is a scalar representing the risk-free rate.

$\mathbf{1}_{\boldsymbol{N}}$ represents a vector of ones of length $N$ : $\left(1_{1}, 1_{2}, \ldots, 1_{i}, \ldots, 1_{N}\right)^{\top}$. 
$x$ is a $N \times 1$ vector of the weights: $\left(x_{1}, x_{2}, \ldots, x_{i}, \ldots, x_{N}\right)^{\top}$.

$M$ is a scalar representing the window size for moving-window estimations.

\subsection{Parameter-Estimation}

The parameters for the respective strategies are not known a priori. In order to implement them, estimators are necessary, which are described in this section. The window size is important, as all parameters are estimated on a rolling-window basis. That means new information is continuously included into the parameters and data older than $M$ is dropped out of the estimation. The abbreviation in the brackets will be used in the empirical section to denote which estimator was used. The estimators are dependent on time, that is for every point of time $t$ a parameter is estimated based on the respective window.

\subsubsection{Arithmetic Mean (AM)}

The first parameter refers to the unknown $\mu$ which represents a vector of expected returns $\left(\mu_{1}, \ldots, \mu_{i}, \ldots, \mu_{N}\right)^{\top}$ and has to be estimated for every time $t$. Here, this will be the arithmetic mean, described by the following formula:

$$
\hat{\mu}_{i, t}=\frac{1}{M} \sum_{j=t-M}^{t-1} r_{i, j}
$$

There, $i$ corresponds to asset $i$ of the set, $t$ to a time, $r$ is the realized return.

\subsubsection{Geometric Mean (GM)}

However, the arithmetic mean might not be suitable as an estimator for mean growth rates. By definition of equation (1) returns are growth rates of prices. Thus, this thesis includes the geometric mean as another estimator, especially as prices follow a geometric series. Furthermore, it is likely that the geometric mean is a more conservative estimator and therefore might lead to better results. The assumption that it is more conservative comes from the fact that for positive real numbers it can be proven that the geometric mean is never greater than the arithmetic mean of the same sample. Furthermore, Jacquier et al. (2003) showed that compounding the arithmetic average is an upwardly biased estimator. The following formula represents the computation of the geometric mean:

$$
\hat{\mu}_{i, t}=\sqrt[M]{\prod_{j=t-M}^{t-1}\left(1+r_{i, j}\right)}-1
$$


Again, $i$ corresponds to asset $i$ of the set, $t$ to a time, $r$ is the realized return and $M$ the window size.

\subsubsection{Variance-Covariance-Matrix (AM/GM)}

Besides the parameter $\mu$ the variance-covariance matrix $\Sigma$ is used. It is a matrix in which the element $\left(e_{i j}\right)$ corresponds to the covariance of the $\mathrm{i}$-th asset with the $\mathrm{j}$-th asset. The diagonal elements $\left(e_{i i}\right)$ are the variance of the $\mathrm{i}$-th asset. As with the mean, the true value is not known which is the reason an estimator has to be provided. In this subsection the usual variancecovariance matrix is described. The estimator of element $\left(e_{i j}\right)$ can be calculated in the following way:

$$
\hat{e}_{i j, t}=\frac{1}{M-1} \sum_{h=t-M}^{t-1} \sum_{k=t-M}^{t-1}\left(r_{i, h}-\hat{\mu}_{i, t}\right)\left(r_{j, k}-\hat{\mu}_{j, t}\right)
$$

The respective window size $M$ will always be the same as for the $\mu$ estimator here. Thus, this results in the usual sample variance-covariance-matrix, which takes the following form:

$$
\hat{\Sigma}_{t}^{u}=\left[\hat{e}_{i j, t}\right]
$$

The " $u$ " as an exponent is for the purpose of assigning a name (" $u$ " stands for usual).

\subsubsection{Bayes-Stein Shrinkage Estimator (BS)}

Jorion (1986) states that usually the portfolio analysis, especially in the MPT framework, is separated into two steps. In the first, the moments and other necessary parameters are estimated and in the second step these are plugged into the optimization as these were the true values. $\mathrm{He}$ argues that such a separation impedes the portfolio analysis through estimation error, especially with the first moment, as the variance seems to be more robust when the sample gets larger. Thus, the Bayesian approach is meant to minimize utility loss coming from the use of sample estimates. It shrinks the sample mean towards a common value, which is in his case the mean of the global minimum variance portfolio. Jorion (1986) showed in a simulation analysis that his shrinkage procedure reduces estimation error significantly.

Following Stein (1955), James and Stein (1961), Jorion (1986) and DeMiguel et al. (2009), the 
equations take the following form:

$$
\begin{aligned}
\hat{\mu}_{t}^{b s} & =\left(1-\hat{\phi}_{t}\right) \hat{\mu}_{t}+\hat{\phi}_{t} \hat{\mu}_{t}^{\text {min }} \\
\hat{\phi}_{t} & =\frac{N+2}{(N+2)+M\left(\hat{\mu}_{t}-\mu_{t}^{\text {min }}\right) \hat{\Sigma}_{t}^{-1}\left(\hat{\mu}_{t}-\mu_{t}^{\text {min }}\right)} \\
0 & <\hat{\phi}_{t}<1 \\
\hat{\Sigma}_{t} & =\frac{M-1}{M-N-2} \hat{\Sigma}_{t}^{u}
\end{aligned}
$$

Again, $M$ represents the window size. $\hat{\mu}_{t}^{\min }$ is the estimated return of the Global Minimum Variance portfolio.

Furthermore, Jorion (1986) provides an estimator for the variance-covariance-matrix:

$$
\hat{\Sigma}_{t}^{b s}=\hat{\Sigma}_{t}\left(1+\frac{1}{M+\lambda}\right)+\frac{\lambda}{M(M+1+\lambda)} \frac{\mathbf{1}_{N} \mathbf{1}_{N^{\top}}}{\mathbf{1}_{N^{\top}} \hat{\Sigma}_{t}^{-1} \mathbf{1}_{\boldsymbol{N}}}
$$

In that formula, $\lambda$ denotes the prior precision.

\subsection{Portfolio Optimization}

In this subsection the different allocation strategies are discussed. For better readability the time index has been omitted. However, it should be noted that for each rebalancing the weights and necessary parameters are estimated. Thus, it is $x_{t}, \mu_{t}$ and $\Sigma_{t}$ everywhere in this subsection. In the empirical section, the rebalancing has been conducted daily. If not stated otherwise explicitly, shortselling and leverage are allowed. That means, an investor can sell assets she does not own (shortselling) or borrow money to buy more assets than she could do with her own wealth (leverage, for example a weight of over $100 \%$ on an asset), see Elton et al. (2003).

\subsubsection{Equally Weighted (Naive)}

The equally weighted portfolio (Naive Portfolio) is one of the easiest strategies for an investor. The idea is to assign the same weight to each asset in a portfolio (e.g.: $20 \%$ for each of 5 stocks in a portfolio). Its desirability comes from the fact that it is easy to implement and non-parametric, it is diversified and also has little to no trading costs, see DeMiguel et al. (2009). This approach is described by the following formula:

$$
x_{i}^{E W}=\frac{1}{N} \quad \forall i \in[1, N]
$$

In this equation $i$ is the index for the $\mathrm{i}$-th asset.

This approach will serve as a benchmark in the empirical section. 


\subsubsection{Mean-Variance (Modern Portfolio Theory - MPT)}

The MPT was one of the milestones in the history of financial economics, founded by the work of nobel prize laureate Harry Markowitz in 1952 with his work Markowitz (1952). Today, different variations of strategies in this framework exist, but all of them share the property of optimization along the efficient frontier. It is the line of sets which are at the same time feasible and optimal. Portfolios below the frontier are feasible but not optimal because there exist combinations which have at least the same return with lower risk or the same level of risk but grant higher return. Portfolios above the efficient frontier are not feasible, see Markowitz (1952) and Elton et al. (2003).

\subsubsection{Target-Return}

This approach is the central idea of the MPT, see Markowitz (1952).

$$
\begin{array}{ll}
\min _{x} & x^{\top} \Sigma x \\
\text { s.t. } & x^{\top} \mu=\mu^{\text {target }} \\
\text { s.t. } & x^{\top} \mathbf{1}_{N}=1
\end{array}
$$

By formulating the Lagrangian of this problem the following solution can be obtained, see Lai et al. (2011) and Jiao (2003):

$$
\begin{aligned}
x^{M V} & =\Sigma^{-1}\left(\lambda_{1} \mu+\lambda_{2} \mathbf{1}_{N}\right) \\
\lambda_{1} & =\frac{a \mu^{\text {target }}-b}{a c-b} \\
\lambda_{2} & =\frac{c-b \mu^{\text {target }}}{d} \\
a & =\mathbf{1}_{N^{\top} \Sigma^{-1} \mathbf{1}_{N}} \\
b & =\mathbf{1}_{N^{\top} \Sigma^{-1} \mu} \\
c & =\mu^{\top} \Sigma^{-1} \mu \\
d & =a c-b^{2}
\end{aligned}
$$

\subsubsection{Sharpe-Ratio-Maximization (Tangency Portfolio)}

Another version within the MPT-family is the Sharpe Ratio, named after William Sharpe who introduced this measurement to compare performances of funds, see Sharpe (1966). However, if 
the Sharpe Ratio is used as a measurement of performance of a portfolio, one can also use it as a basis to develop a strategy. This is also known as the tangency portfolio, which aims to maximize the proportion of portfolio return per unit of portfolio risk. Thus, it is expressed in the following way:

$$
\begin{array}{ll}
\max _{x} & \frac{x^{\top}\left(\mu-r_{f}\right)}{x^{\top} \sum x} \\
\text { s.t. } & x^{\top} \mathbf{1}_{N}=1
\end{array}
$$

According to Elton et al. (2003) the solution takes the following form:

$$
\begin{aligned}
x^{S R} & =\frac{\Sigma^{-1}\left(\mu-r_{f}\right)}{\mathbf{1}_{N^{\top} \Sigma^{-1}\left(\mu-r_{f}\right)}} \\
x^{S R} & =\frac{\Sigma^{-1} \mu}{\mathbf{1}_{N^{\top} \Sigma^{-1} \mu}}
\end{aligned}
$$

It can be seen that 22 follows from 21 when the risk-free rate is set to zero, which is the same as maximizing the Certainty Equivalent.

\subsubsection{Certainty Equivalent-Maximization}

Similar to the Sharpe Ratio the Certainty Equivalent serves as a measurement for portfolio performance. This metric can be interpreted as the return an investor would require from a risk-free asset to be indifferent between the risk-free asset and the portfolio. Together with the Sharpe Ratio it shares the property of penalizing dispersion, however it uses an additive connection instead of a multiplicative one. The maximization problem is denoted as follows, see DeMiguel et al. (2009):

$$
\begin{array}{ll}
\max _{x} & x^{\top} \mu-\frac{\gamma}{2} x^{\top} \Sigma x \\
\text { s.t. } & x^{\top} \mathbf{1}_{N}=1
\end{array}
$$

Here, $\gamma$ is a parameter representing the risk aversion of the investor. DeMiguel et al. (2009) also provide a solution which can be obtained by taking the first derivative with respect to and solving for $x$.

$$
x^{C E}=\frac{\Sigma^{-1} \mu}{\mathbf{1}_{N^{\top} \Sigma^{-1} \mu}}
$$

It can be seen that this solution is identical to the one of the Sharpe-Ratio-Maximization before, thus the results for the Certainty-Equivalent-Maximization will not be reported in the empirical section. 


\subsubsection{Global Minimum Variance}

This strategy is also an option of the MPT and can be seen as a special case of mean-variance, as mean is ignored completely. This is the same as assuming that all means are equal (e.g.: $\left.\mathbf{1}_{N}=\mu\right)$, see DeMiguel et al. (2009):

$$
\begin{array}{ll}
\min _{x} & x^{\top} \Sigma x \\
\text { s.t. } & x^{\top} \mathbf{1}_{N}=1
\end{array}
$$

By formulating the Lagrangian, Clarke et al. (2013) provide a solution to this problem:

$$
x^{G M V}=\frac{\Sigma^{-1} \mathbf{1}_{N}}{\mathbf{1}_{N^{\top} \Sigma^{-1} \mathbf{1}_{N}}}
$$

From a theoretical point of view this approach should be more risk-averse than the classical MPT approach which is bound to a target return. This assumption is reasoned by the positive correlation of risk and return. As a minimum return is not required in the Global Minimum Variance strategy, a minimum level of risk is not necessary to be taken by an investor. Thus, it is the lowest point on the efficient frontier, see Elton et al. (2003).

\subsubsection{Geometric Mean Maximization}

In contrast to the MPT-family the maximization of the geometric mean is a dynamic approach. Whereas the Mean-Variance strategies are static in the sense that they only take one future period into account, the GMM considers a large amount of periods. The original idea was that, when MPT results in a set of efficient portfolios, an investor still has to choose one among them. This model shall help to determine a portfolio of the feasible sets which maximizes the terminal wealth. Furthermore, to achieve the maximum terminal wealth, it was argued that the growth rate of wealth should be maximized, which is the geometric mean of the portfolio return in such a multi-period model, see Estrada (2010). The mathematical formulation takes the following form:

$$
\begin{array}{ll}
\max _{x} & \sqrt[L]{\prod_{t=1}^{L} 1+x_{t}^{\top} \mu_{t}-1} \\
\text { s.t. } & x^{\top} \mathbf{1}_{\boldsymbol{N}}=1
\end{array}
$$

In the equation above, $L$ represents the last considered period. It is possible to have a finite $L$ as well as $\lim _{L \rightarrow \infty}$. As Estrada (2010) shows, a Taylor-Expansion of second order leads to the 
following approximation:

$$
\begin{array}{ll}
\max _{x} & \exp \left\{\ln \left(1+x^{\top} \mu\right)-\frac{x^{\top} \Sigma x}{2\left(1+x^{\top} \mu\right)^{2}}\right\}-1 \\
\text { s.t. } & x^{\top} \mathbf{1}_{\boldsymbol{N}}=1
\end{array}
$$

As the problem above is not solvable analytically, numerical methods will be used for the empirical study. In order to find a solution for equation 28 the Nelder-Mead algorithm is used, see Nelder and Mead (1965). Furthermore, for the empirical part, a short-constrained version of the problem is maximized as well. That means, that additional constraints are imposed where every weight $x$ has to be non-negative. For this problem the L-BFGS-B algorithm is used, see Byrd et al. (1995).

\subsubsection{Conditional Value-at-Risk Minimization (CVaR)}

Usually, investors want to control the risks of their portfolio. However, the strategies of the MPT-family include risk only in terms of expected portfolio variance. Krokhmal et al. (2003) note that often distributions such as normal- or log-normal-distribution are assumed. However, such assumptions about the distribution are contrary to stylized facts as returns usually exhibit heavy tails, see Petukhina et al. (2018). A first step towards better risk management was the introduction of the "Value-at-Risk" ( $\mathrm{VaR})$. The $\mathrm{VaR}$ represents a specific $\alpha$-quantile of the return distribution. In other words, the $\mathrm{VaR}$ is the value, which will not be underrun with a likelihood of $1-\alpha$. However, in its application problems such as instability occur and it does not work well numerically for distributions other than normal ones. Furthermore, it is no coherent risk measure as it fails to satisfy the subadditivity property, see Artzner (1999). Thus, the Conditional Valueat-Risk ( CVaR) was introduced, which is coherent and also delivers more information. Whereas $\mathrm{VaR}$ only defines a tail, CVaR describes what happens within this tail. In other words, CVaR is the expected value when a tail event occurs, which is the reason sometimes it is called "Expected Shortfall". Furthermore, the CVaR is not reliant on stylized estimators coming from a normal distribution, as it includes higher moments and takes the actual distribution into account. With respect to Rockafellar and Uryasev (2000) and Petukhina et al. (2018) the following problem has to be solved to find a CVaR-optimal portfolio:

$$
\begin{array}{ll}
\min _{x} & C V a R_{\alpha}(x) \\
\text { s.t. } & x^{\top} \mathbf{1}_{N}=1 \\
\text { s.t. } & x_{i} \geq 0
\end{array}
$$


Where

$$
C \operatorname{VaR}_{\alpha}(x)=-\frac{1}{1-\alpha} \int_{x^{\top} \mu \leq-\operatorname{VaR}_{\alpha}(x)} x^{\top} \mu f\left(x^{\top} \mu \mid x\right) \mathrm{d} x^{\top} \mu
$$

As stated above, $\operatorname{VaR}_{\alpha}(x)$ is the respective $\alpha$-quantile of the return distribution. The term $f\left(x^{\top} \mu \mid x\right)$ represents the probability density function of the portfolio return dependent on the weights $x$. It should be noted that it is possible to impose additional constraints, as for example a target return which shall be reached at least by the portfolio, compare with Petukhina et al. (2018). However, an analytical solution is not possible for the problem. Additionally, the a priori distribution is not known and thus is estimated using the empirical cumulative counterpart based on the window $M$.

In order to find the optimal weights to minimize CVaR a simulation is done, where a large set of weights is drawn from the uniform distribution. That is, $\eta$ sets with $N$ independently weights from the continuous uniform distribution $U(0,1)$ are created. These simulated set of weights is then used to calculate a large set of historical portfolio returns, from which the empirical cumulative distribution function is estimated. In the next step, the simulation finds the subset of weights which has the smallest CVaR among all simulated portfolios.

\subsubsection{LIBRO}

All strategies mentioned before assume that an investor can buy or sell any quantity at any time. However, this might not reflect the reality, where trading depends on supply and demand on the markets. Thus, Trimborn et al. (2019) proposed a portfolio strategy which controls for the liquidity aspect. The idea behind this is to create an upper boundary for each weight, dependent on the liquidity the respective asset has, which is imposed as an additional constraint. Formally, this approach is expressed as follows:

$$
x_{i} \leq \frac{T V_{i} f_{i}}{W}
$$

There, $i$ stands for the i-th asset, $T V$ represents the sample median trading volume as a proxy for liquidity, $f$ the speed an investor intends to clear the current position and $W$ the wealth of the investor.

The beauty of this strategy lies in its simplicity and universality, as it can be implemented easily into any other strategy. In this research it will be added to the Mean-Variance strategy of equation 12 and to the CVaR strategy of equation 29. To solve the equations when the LIBRO constraint is added to the MPT approach, it appears to be a quadratic programming problem. To solve it the quadprog-package in $\mathrm{R}$ is used, see Goldfarb and Idnani (1983). To find the optimal CVaR-LIBRO strategy a simulation is done similar to the original $\mathrm{CVaR}$ before. 


\subsection{Performance Metrics}

In order to assess and compare the performance of the different strategies it is necessary to introduce comparable metrics. This section deals with the different measurements used to evaluate the portfolios described before. In the following let $\hat{\Psi}$ denote the estimator for success measurement. $\hat{\mu}_{k}$ and $\hat{\sigma}_{k}^{2}$ denote the arithmetic mean return and variance of the respective k-th strategy.

\subsubsection{Certainty Equivalent}

The Certainty Equivalent was already mentioned in the Mean-Variance section. As said there, it gives information about the rate a risk-free asset must return at least, such that an investor was indifferent between the respective $\mathrm{k}$-th portfolio and the risk-free asset.

Following DeMiguel et al. (2009) and Petukhina et al. (2018), the Certainty Equivalent takes the form:

$$
\hat{\Psi}_{k, \gamma}^{c e q}=\hat{\mu}_{k}-\frac{\gamma}{2} \hat{\sigma}_{k}^{2}
$$

There, $\gamma$ denotes the risk aversion of an investor.

\subsection{2 (Adjusted) Sharpe Ratio}

The Sharpe Ratio, also mentioned in the Mean-Variance section before, was named after William F. Sharpe who referred to this as "Return-to-Variability"-ratio, see Sharpe (1966). Economically, it can be interpreted as how much return an investor receives per unit of risk. Said colloquially, it is how much return $\mu$ an investor could "buy" paying an additional unit of risk $\sigma$. Formally, it is defined as:

$$
\hat{\Psi}_{k}^{s r}=\frac{\hat{\mu}_{k}}{\sqrt{\hat{\sigma}_{k}^{2}}}
$$

However, investors might be interested in skewness and kurtosis as well, thus, to assess the performance properly, Pézier and White (2008) proposed the Adjusted Sharpe Ratio:

$$
\hat{\Psi}_{k}^{a s r}=\hat{\Psi}_{k}^{s r}\left[1+\left(\frac{\hat{S}}{6}\right) \hat{\Psi}_{k}^{s r}-\left(\frac{\hat{K}}{24}\right)\left(\hat{\Psi}_{k}^{s r}\right)^{2}\right]
$$

In this formula, $\hat{S}$ and $\hat{K}$ represent sample skewness and sample excess kurtosis. Following Petukhina et al. (2018), this measurement incorporates the preference for positive skewness and negative excess kurtosis, as it penalizes the respective opposite. This is important, as a distribution with negative skewness and positive excess kurtosis increases tail risks of a portfolio. These are not desired by investors. 


\subsubsection{Turnover}

Another important factor to assess performance of a portfolio is the amount of trading necessary to implement a strategy. This is of importance because it is directly related to costs of a strategy, as in reality an investor usually has to pay for her transactions.

Following DeMiguel et al. (2009) and Petukhina et al. (2018), the following computation will be used:

$$
\hat{\Psi}_{k}^{t o}=\frac{1}{T-M} \sum_{t=M+1}^{T} \sum_{i=1}^{N}\left(\left|\hat{x}_{k, i, t+1}-\hat{x}_{k, i, t+}\right|\right)
$$

Here, $\hat{x}$ denotes the weight on asset $i$, in time $t+1$ after rebalancing and right before rebalancing $(t+) . k$ denotes the $\mathrm{k}$-th strategy. It can be seen that this formula calculates the absolute sum of changes in the weights, so the interpretation is that the larger this metric is, the higher the implementation cost of the strategy. Thus, the smaller this metric, the better the strategy with respect to this measurement.

\subsubsection{Terminal Return}

The last metric used is the terminal return, sometimes also called terminal wealth. It is an important factor, because it denotes the final outcome of a strategy at the end of the investing period. It does not control for risk in any way. The importance of this metric lies in the fact, that for example a funds managers performance may be measured in the wealth she created for her investors. The following formula is used for the computation:

$$
\hat{\Psi}_{k}^{t r}=\prod_{t=M+1}^{T}\left(1+r_{t, k}\right)
$$

Here, $r$ denotes the realized portfolio return, $k$ the k-strategy and $t$ is the time index. As before, $M$ is the window size, however this formula is not based on a rolling window, rather it starts after the first window, when the first portfolio results can be computed. This formula thus represents cumulative performance at the final time $T$ and can also be used to calculate the daily compound return of a portfolio.

\subsection{Significance}

In order to compare the results, it is not only necessary to look at the metrics themselves but also whether they are significant. As for example the Certainty Equivalent relies on the first moment of the final portfolios, these will be compared and tested for significant differences. As the naive 
portfolio serves as a benchmark in this paper, a classical one sample t-test is conducted. The formula for the t-statistics is:

$$
t\left(\hat{\mu}_{k}\right)=\frac{\hat{\mu}_{k}-\mu_{0}}{\sqrt{\hat{\sigma}_{k}^{2}}} \sqrt{n}
$$

In the formula above, $n$ denotes the number of observations, thus is equal to $T-M$, where $M$ is the window size and $T$ the total number of observations. $\mu_{0}$ is the benchmark mean, thus the mean of the naive portfolio. If the null hypothesis is true this statistics is t-distributed with $n-1$ degrees of freedom, where the hypotheses are specified as follows, see Toutenburg and Heumann (2008):

$$
\begin{aligned}
& H_{0}: \hat{\mu}_{k}=\mu_{0} \\
& H_{1}: \hat{\mu}_{k} \neq \mu_{0}
\end{aligned}
$$

The null hypothesis $H_{0}$ is rejected if $\left|t\left(\hat{\mu}_{k}\right)\right|>t_{n-1 ; 1-\frac{\alpha}{2}}$ where $t_{n-1 ; 1-\frac{\alpha}{2}}$ is the $1-\frac{\alpha}{2}$ quantile of the t-distribution with $n-1$ degrees of freedom. The respective p-value denotes the likelihood that such a value is reached under the null hypothesis. However, the test requires that the random variable, in this case $\hat{\mu}_{k}$, shall be normally distributed. To assess whether the portfolios follow a normal distribution a Shapiro-Wilk test is conducted for each of them, as it delivers the greatest power among normality tests, see Razali and Yap (2011). However, Stonehouse and Forrester (1998) demonstrated that the t-test is robust against violations of the normality assumption, especially if the skewness is not too extreme. To interpret the sample skewness $\hat{S}$ a rule-of-thumb is used, see Bulmer (1979). By this rule-of-thumb a distribution is approximately symmetric when $|\hat{S}| \leq 0.5$, it is moderately skewed when $0.5 \leq|\hat{S}| \leq 1$ and highly skewed when $1 \leq|\hat{S}|$.

One may argue that a two sample test is more appropriate, such as the Welch-test, however as the strategies are compared with a benchmark, this "benchmark-mean" is considered as an externality. Furthermore, Stonehouse and Forrester (1998) also showed that the Welch-test is not robust against violations of normality.

\section{Empirical Analysis}

In this section the empirical results of the strategies discussed before are analyzed. The nonparametric Naive Portfolio serves as a benchmark here, as the main question is whether an approach can outperform such a simple asset allocation but also whether it can do it efficiently. That means, the performance has to be significantly different such that it is worth the effort a potential investor has to make in order to implement the respective model. For all parametric 
strategies three different estimators are used. These are denoted by the abbreviation in the brackets, where (AM) stands for the arithmetic mean, (GM) for the geometric mean and (BS) for the Bayes-Stein estimators. The (AM) and (GM) strategies both use the same Variance-Covariance matrix and only differ in the mean. The (BS) portfolios have their own first and second moments as inputs, see section 3.1 .4 .

Besides discussing the strategies themselves, this section also deals with the question whether the usage of different estimators has some impact on the performance of the portfolios.

First of all, values for the other parameters, which were used in the methodological section, need to be assigned. The following table shows the values used.

\begin{tabular}{l|c|l}
\hline \hline Parameter & Description & Value \\
\hline$r_{f}$ & Risk-Free Rate & 0 \\
$\mu^{\text {target }}$ & Target Return (MPT) & $2.6 * 10^{-4}$ \\
$M$ & Window Size & 120 \\
$\mathrm{~W}$ & Wealth (LIBRO) & 1 \\
$f$ & Clear Speed (LIBRO) & $10^{-4}$ \\
$\gamma$ & Risk Aversion & 1 \\
$\alpha$ & Risk-Quantile & 0.05 \\
$\eta$ & Number of Simulations (CVaR) & $1.5 * 10^{4}$ \\
$\lambda$ & Prior Precision (BS) & 1 \\
\hline \hline
\end{tabular}

The chosen target return might seem very low, but the observations and thus the calculated (expected) returns are daily. The denoted return above corresponds to an approximate return of $10 \%$ per year.

The portfolios are considered as indices in this thesis. That is, for each period $t$ the optimal weights $x$ are calculated for each strategy and then multiplied with the true returns $r$. In matrix notation this is written as $x^{\top} r$. The fictional investor starts with a wealth of 1 and has neither liquidity constraints nor is she already invested. Then for the next period $t+1$ the same procedure happens. This could be considered as an investor who sells everything after $t$, thus realizes the return $x^{\top} r$ and then uses her wealth of $1+x^{\top} r$ to invest with the new weights.

Another problem which occured was that for some of the assets data of their trading volume was not available. In order to use the LIBRO-strategy on these assets anyway, the missing values were resampled in the following way: As for every asset with non-missing values the sample median trading volume on the window size was calculated, this results in a vector of median sample $T V \mathrm{~s}$ 
in every $t$. Let this vector be denoted with $\zeta$, then instead of taking missing values these NAs were replaced with $\min (\zeta)$.

The abbreviations "CEQ", "SR", "ASR", "TO" and "TR" refer to the metrics Certainty Equiv-

\begin{tabular}{rrrrrr}
\hline \hline & CEQ & SR & ASR & TO & TR \\
\hline Naive & 0.0008 & 0.0898 & 0.0895 & 0.0000 & 1.8025 \\
Constrained Minimum Variance (AM) & 0.0000 & 0.0077 & 0.0077 & 0.3171 & 1.0213 \\
Global Minimum Variance (AM) & 0.0000 & 0.0090 & 0.0090 & 0.3013 & 1.0267 \\
Sharpe Ratio (AM) & -0.9658 & -0.0435 & -0.0486 & 80.4674 & -0.2901 \\
F-Geometric Mean Maximization (AM) & 0.0008 & 0.0915 & 0.0913 & 0.0114 & 1.8323 \\
C-Geometric Mean Maximization (AM) & 0.0006 & 0.0648 & 0.0646 & 0.2257 & 1.5871 \\
LIBRO-MPT (AM) & 0.0001 & 0.0131 & 0.0131 & 0.3211 & 1.0449 \\
LIBRO-CVaR (AM) & 0.0010 & 0.0976 & 0.0976 & 0.6700 & 2.1400 \\
CVaR (AM) & 0.0009 & 0.0849 & 0.0850 & 0.6699 & 1.9232 \\
Constrained Minimum Variance (GM) & 0.0000 & 0.0076 & 0.0076 & 0.3180 & 1.0209 \\
Global Minimum Variance (GM) & 0.0000 & 0.0090 & 0.0090 & 0.3013 & 1.0267 \\
Sharpe Ratio (GM) & -0.4409 & -0.0261 & -0.0283 & 65.2649 & 0.0172 \\
F-Geometric Mean Maximization (GM) & 0.0008 & 0.0910 & 0.0908 & 0.0120 & 1.8242 \\
C-Geometric Mean Maximization (GM) & 0.0005 & 0.0632 & 0.0629 & 0.2165 & 1.5122 \\
LIBRO-MPT (GM) & 0.0001 & 0.0130 & 0.0130 & 0.3206 & 1.0444 \\
LIBRO-CVaR (GM) & 0.0010 & 0.0996 & 0.0995 & 0.6722 & 2.1757 \\
CVaR (GM) & 0.0009 & 0.0876 & 0.0881 & 0.6731 & 1.9805 \\
LIBRO-CVaR (BS) & 0.0010 & 0.0969 & 0.0977 & 0.6744 & 2.1588 \\
CVaR (BS) & 0.0010 & 0.0962 & 0.0961 & 0.6709 & 2.1160 \\
\hline F-Geometric Mean Maximization (BS) & 0.0008 & 0.0908 & 0.0906 & 0.0120 & 1.8225 \\
C-Geometric Mean Maximization (BS) & 0.0002 & 0.0228 & 0.0227 & 0.1990 & 1.1287 \\
Constrained Minimum Variance (BS) & 0.0000 & 0.0080 & 0.0080 & 0.3924 & 1.0232 \\
Global Minimum Variance (BS) & 0.0000 & 0.0090 & 0.0090 & 0.3013 & 1.0267 \\
Sharpe Ratio (BS) & -0.1525 & -0.0426 & -0.0473 & 32.5545 & 1.1020 \\
\hline
\end{tabular}

Table 1: Values of Success Metrics for all Strategies a EMAIS_Visual

alent, Sharpe Ratio, Adjusted Sharpe Ratio, Turnover and Terminal Return. It can be seen in table 1 that the Global Minimum Variance strategy and the Constrained Minimum Variance strategy have a Certainty Equivalent of approximately zero for all three estimators. Furthermore, the 
LIBRO-MPT is only slightly above with a CEQ of approximately $0.01 \%$. Interestingly, the Naive Portfolio reports a CEQ of $0.08 \%$, which is only exceeded by the three CVaR and LIBRO-CVaR strategies. Additionally, it is interesting that the three Sharpe Ratio strategies are the only ones to report a negative $C E Q$ and indeed very extreme values. The interpretation of for example the -0.9658 of the Sharpe Ratio (AM) is that if the risk-free rate $r_{f}$ is greater than $-96.58 \%$ an investor would choose the risk-free asset over this portfolio. However, it is already known that MPT strategies tend to extreme weights and outlying results, see DeMiguel et al. (2009). Nonetheless, the Sharpe Ratio approach is the only one where this thesis reports extreme results, which can also be seen in the other metrics, as for example the turnover which is around several thousand times higher than for the other portfolios. Yet, the rest of the MPT strategies has moderate results compared to the Sharpe-Ratio-Maximization.

In terms of (Adjusted) Sharpe Ratio not only the three LIBRO-CVaR strategies and the BayesStein CVaR outperform the Naive Portfolio, but also the three unconstrained Geometric Mean Maximization approaches do so. For example, an investor would get approximately 1 percentage point more return per unit of risk when choosing the LIBRO-CVaR instead of the Naive Portfolio.

Interestingly, these strategies which have performed so well with respect to the metrics before do not perform good in terms of turnover. In fact, the six CVaR strategies are the worst, except the Sharpe Ratio strategies which again report extreme values. Obviously, the Naive Portfolio has the best turnover with a true zero, as no changes in relative weights occur. However, in reality that turnover would not be zero, as an investor has to rebalance in order to keep the relative weights constant. To illustrate that, imagine a portfolio of two assets $\left(y_{1}, y_{2}\right)$ at an arbitrary time $t$ with value $(1,1)$. Then at time $t+1$ one asset loses $20 \%$ and the other one gains $20 \%$. Now, the total value of the portfolio is still 2 , but the relative weights have shifted from $50 \%$ on both to $40 \%$ and $60 \%$. Thus, the investor must sell a littlebit of $y_{2}$ and buy a littlebit of $y_{1}$ to keep the relative weights constant. However, it can be assumed that the turnover of the Naive Portfolio still would be small as returns are typically small as well. Furthermore, the unconstrained Geometric Mean Portfolios perform best by far, which is surprising as well. A possible reason for this is, that these consider a large amount of future periods and try to optimizie with respect to all of them. Thus, less rebalancing could be necessary. Yet, this cannot be said surely.

The last metric reported in the table above is the terminal return. As mentioned in section 3 , it gives information about the total wealth accumulated at the end. Again, the best performing 
strategies are the three LIBRO-CVaR approaches with a terminal return of circa $210 \%$, closely followed by the normal CVaR strategies with circa 200\%. Thereafter, the unconstrained Geometric Mean Maximization and the Naive Portfolio are pretty close, reporting circa $180 \%$, and then most of the strategies take values somewhere between $100 \%$ and $150 \%$. The Sharpe Ratio Portfolios have performed bad again, the best one being the Bayes-Stein estimated with at least positive terminal return. The Tangency Portfolio based on the geometric mean has a terminal return below $100 \%$ and above zero, which is equal to a loss, and the arithmetic mean based Tangency Portfolio has a negative value which can be interpreted as ending up in debt.

With respect to these metrics, the LIBRO-CVaR seems to be the best performing strategy. However, this depends on the associated trading costs, as these three strategies also have the highest turnover and therefore are the most expensive approaches. The unconstrained Geometric Mean Maximization is, besides the Naive Portfolio, the most cost efficient one and as already highlighted, even the Naive Portfolio would have trading costs. Nonetheless, the Geometric Mean approach does not have a significant different arithmetic mean compared to the Naive Portfolio, nor are its metrics far higher or lower. Figure 1 shows the cumulative performance of a few chosen strategies.

Furthermore, it is of interest whether the strategies have a mean significantly different from the benchmark portfolio. As already described, a simple t-test is used in order to test the meandifference. Table 2 denotes the absolute sample skewness, the p-values of the Shapiro-Wilk test of normality and the p-values of the t-test. The sample skewness and Shapiro-Wilk p-values are reported, as the t-test requires normality, but is robust against violations if the skewness is not too extreme, see Stonehouse and Forrester (1998). 


\begin{tabular}{|c|c|c|c|}
\hline & $|\hat{S}|$ & p-value (SW-Test) & $\mathrm{p}$-value (t-Test) \\
\hline Naive & 0.1297 & 0.0000 & $N A$ \\
\hline Constrained Minimum Variance (AM) & 0.8489 & 0.0000 & 0.0002 \\
\hline Global Minimum Variance (AM) & 0.6738 & 0.0000 & 0.0001 \\
\hline Sharpe Ratio (AM) & 22.2189 & 0.0000 & 0.2227 \\
\hline F-Geometric Mean Maximization (AM) & 0.0649 & 0.0000 & 0.9465 \\
\hline C-Geometric Mean Maximization (AM) & 0.1124 & 0.0000 & 0.6697 \\
\hline LIBRO-MPT (AM) & 0.6236 & 0.0000 & 0.0003 \\
\hline LIBRO-CVaR (AM) & 0.0730 & 0.0000 & 0.5354 \\
\hline CVaR (AM) & 0.1583 & 0.0000 & 0.7938 \\
\hline Constrained Minimum Variance (GM) & 0.8484 & 0.0000 & 0.0002 \\
\hline Global Minimum Variance (GM) & 0.6738 & 0.0000 & 0.0001 \\
\hline Sharpe Ratio (GM) & 24.0747 & 0.0000 & 0.4561 \\
\hline F-Geometric Mean Maximization (GM) & 0.0776 & 0.0000 & 0.9608 \\
\hline C-Geometric Mean Maximization (GM) & 0.2980 & 0.0000 & 0.4954 \\
\hline LIBRO-MPT (GM) & 0.6225 & 0.0000 & 0.0003 \\
\hline LIBRO-CVaR (GM) & 0.0218 & 0.0000 & 0.4996 \\
\hline CVaR (GM) & 0.5913 & 0.0000 & 0.7196 \\
\hline Constrained Minimum Variance (BS) & 0.7320 & 0.0000 & 0.0006 \\
\hline Global Minimum Variance (BS) & 0.6738 & 0.0000 & 0.0001 \\
\hline Sharpe Ratio (BS) & 20.8450 & 0.0000 & 0.2219 \\
\hline F-Geometric Mean Maximization (BS) & 0.0653 & 0.0000 & 0.9638 \\
\hline C-Geometric Mean Maximization (BS) & 0.3856 & 0.0000 & 0.0468 \\
\hline LIBRO-MPT (BS) & 0.4591 & 0.0000 & 0.0013 \\
\hline LIBRO-CVaR (BS) & 0.7409 & 0.0000 & 0.5213 \\
\hline $\mathrm{CVaR}(\mathrm{BS})$ & 0.0038 & 0.0000 & 0.5606 \\
\hline
\end{tabular}

Table 2: Absolute Sample Skewness and p-values for Normality and t-Test for all Strategies a. EMAIS_Visual

Given the table it can be seen that the hypothesis of normal distribution has to be rejected in all cases. However, excluding the Sharpe-Ratio-Maximization, for all of the strategies an absolute sample skewness below 1 is reported. That means, following the rule of thumb of Bulmer (1979), the t-test still remains powerful for these, as most of them are only moderately skewed or even approximately symmetric. That the first row in the t-test column of the table contains an $N A$ 
comes from the fact that the Naive Portfolio is the benchmark, thus testing for difference between the strategy and itself again is redundant as they are exactly equal by construction. Thus, only the p-values of the Sharpe Ratio strategies should be treated carefully, as power loss of the t-test could be heavy due to extreme violations of the underlying assumptions. One can see, that regardless of the estimator used, the Constrained Minimum Variance, Global Minimum Variance and LIBRO-MPT are significant at the $1 \%$ level, and except the latter one even at the $0.1 \%$ level. Furthermore, the Constrained Geometric Mean Maximization with Bayes-Stein Estimator is significant at the $5 \%$ level. The rest of the strategies are not even significant on the $20 \%$ level and therefore should be interpreted as not significantly different from the Naive Portfolio. However, these results should not be the nonplusultra when it comes to judging as the t-test uses the arithmetic mean which might not be the best metric for returns. Table 1 presents the performance metrics introduced in section 3 . 


\section{Cumulative Performance}

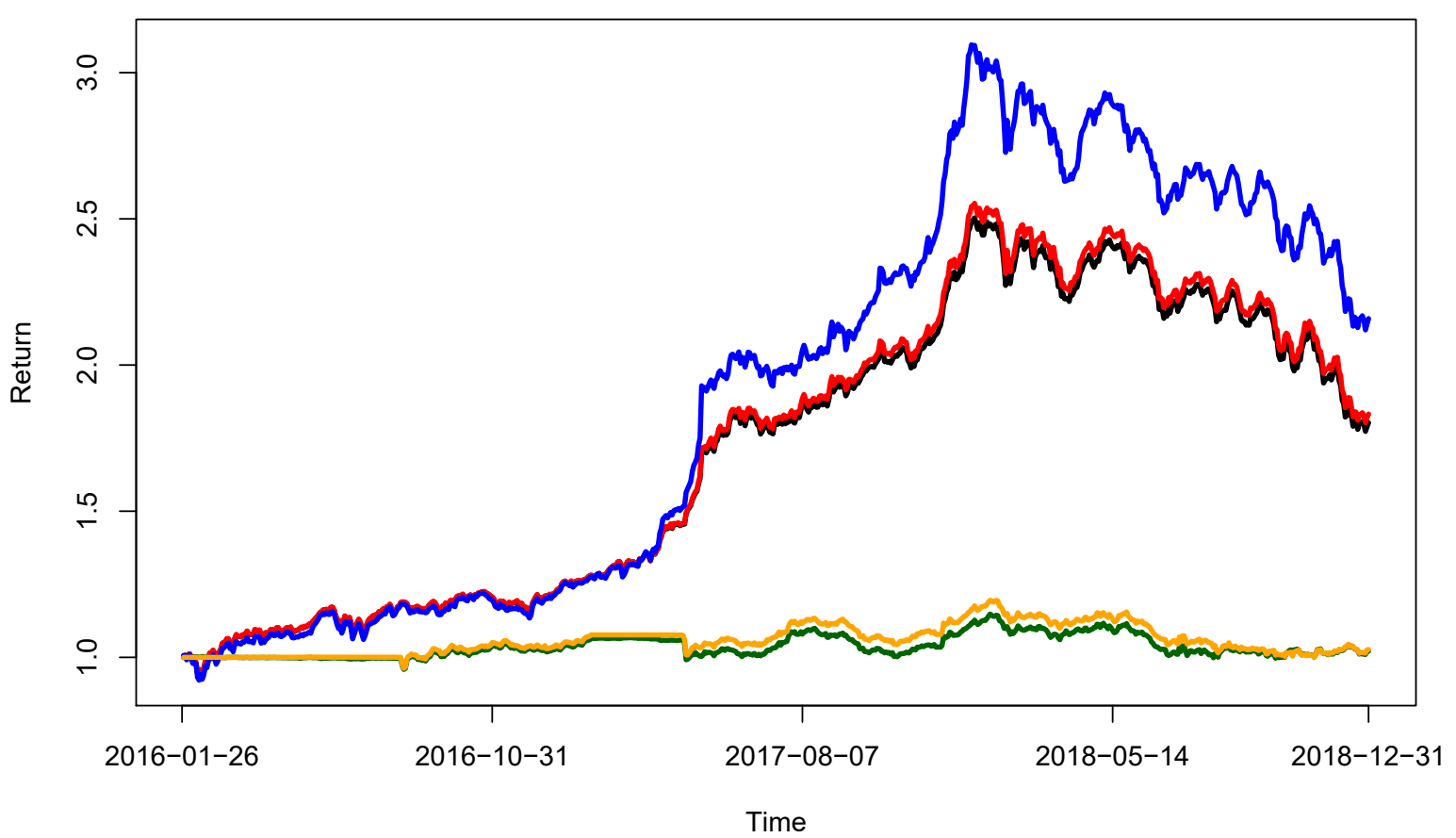

Figure 1: Equally Weighted, Constrained Minimum Variance (AM), Unconstrained Geometric Mean Maximization (AM), LIBRO-CVaR (BS) and Global Minimum Variance (BS) Q EMAIS_Visual

One can see that the Naive Portfolio and the unconstrained Geometric Mean Maximization are approximately equal to each other and so are the Global Minimum Variance strategy and the Constrained Minimum Variance Portfolio. Up to some point, the LIBRO-CVaR Portfolio lies approximately on the Naive Portfolio and then starts outperforming it, ending up with a higher terminal wealth. Given the metrics and the figure above, it should be checked whether the LIBROCVaR portfolio is cost efficient, and if it is not an investor should choose either the Naive Portfolio or one of the Geometric Mean Maximizations. Figure 2 is a $\mu$ - $\sigma$-diagram of the portfolios, using different symbols and colors for strategy and estimator used. The Sharpe Ratio portfolios have been excluded as they contain extreme outliers. As already seen in the tables and figures before, the LIBRO-CVaR and CVaR are the best among those strategies depicted, followed by the unconstrained Geometric Mean Maximization. Furthermore, no clear relationship between performance and estimators can be seen.

Besides from the individual quality, it is also of interest whether the usage of different estimators has impact on the portfolio performance. Figure 3 shows how mean and standard deviation of the 


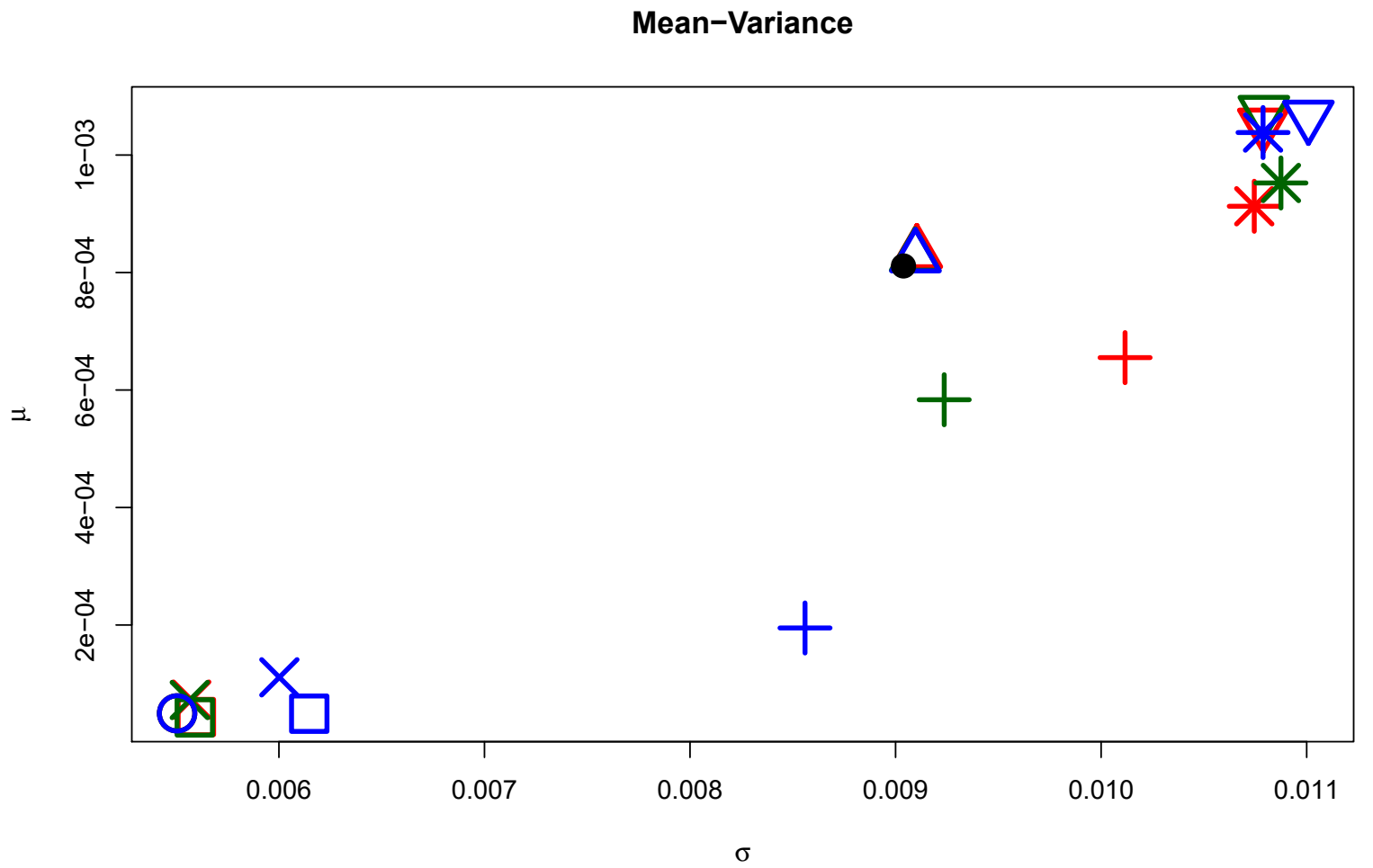

Figure 2: Mean-Variance Diagram of Strategies a EMAIS_Visual

Colors: (AM), (GM), (BS)

Symbols: $\square$ Constrained Minimum Variance, $\bigcirc$ Global Minimum Variance, $\triangle$ Unconstrained Geometric Mean Maximization, + Constrained Geometric Mean Maximization, $\times$ LIBRO-MPT, $\nabla$ LIBRO-CVaR, * CVaR

Black Bullet: • Naive Portfolio

strategies are distributed with respect to their estimator. The Sharpe Ratio Maximizations have been removed from the computation due to their outlyingness. It can be seen that on median the mean return of the Bayes-Stein strategies is far below those of the geometric mean and arithmetic mean, having only approximately one third of it. However, its standard deviation is also slightly lower on median. For both parameters, the range of their distribution is approximately equal for all three estimators. Anyway, the portfolios might contain outliers, thus in the boxplots of figure 4 robust location- and disperion parameter, namely the median and the interquartile range, are depicted. Using robust parameters, the location of returns among strategies has changed. The median of the median return is close to zero for the arithmetic mean estimations as well as for the Bayes-Stein estimation. However, the median return for the geometric mean estimator is even higher than the mean return (ca. 0.001 compared to ca. 0.0006). The median interquartile 

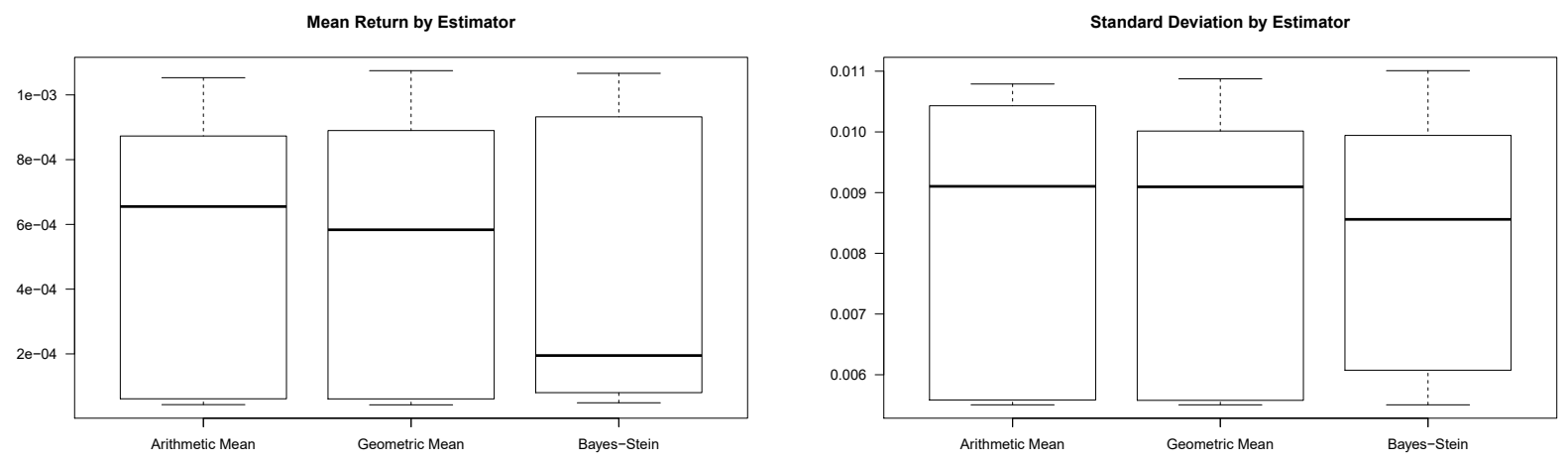

Figure 3: Boxplot of Mean and Standard Deviation of Portfolio Returns by Estimators used a. EMAIS_Visual
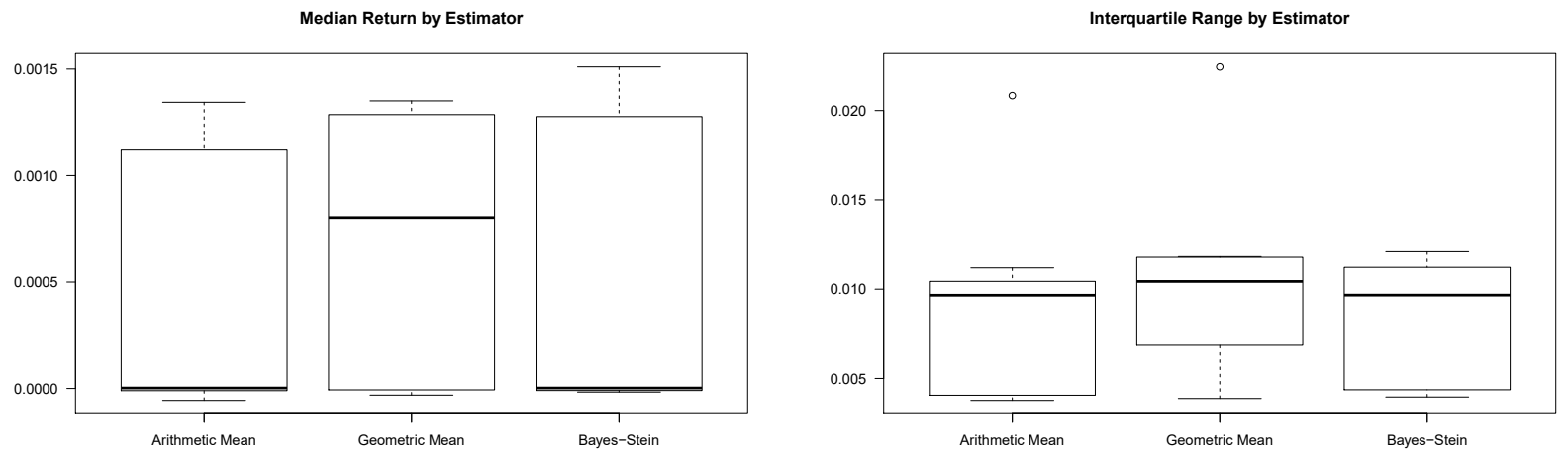

Figure 4: Boxplot of Median and Interquartile Range of Portfolio Returns by Estimators used a. EMAIS_Visual

range is higher than the standard deviation for all three estimators, although the IQR is barely comparable to the standard deviation as they have different interpretations. As pointed out before, the geometric mean might be a better measurement to assess the performance of a portfolio. Furthermore, the final wealth is of interest for an investor, thus these two metrics are shown in the boxplots of figure 5 The results for geometric mean as a location parameter are similar to those of the arithmetic mean. Again, Bayes-Stein comes out with the lowest mean, whereas arithmetic mean strategies and geometric mean strategies have only slightly different median returns. Interestingly, the median of the geometric mean strategies is approximately the same for the three parameters arithmetic mean, median and geometric mean. Given these figures it seems that the portfolios which use the geometric mean as an estimator have stablest performance. When it comes to terminal return, the portfolios which used the arithmetic mean as an input parameter perform slightly better than the geometric mean strategies. The Bayes-Stein portfolios come out 

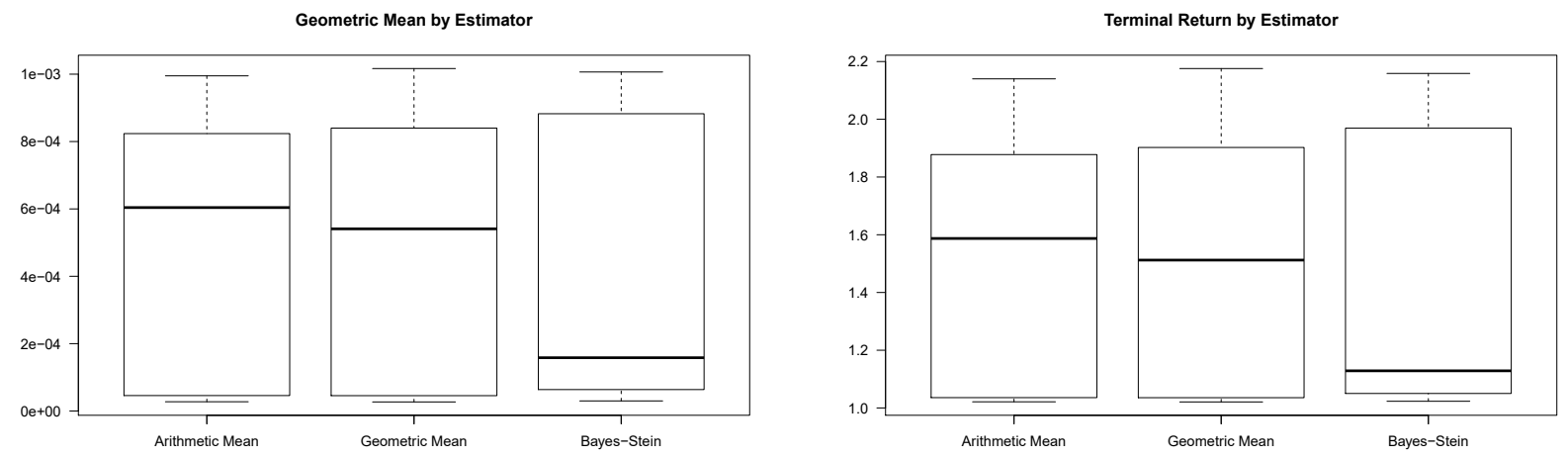

Figure 5: Boxplot of Geometric Mean and Terminal Return of Portfolios by Estimators used a. EMAIS_Visual

with a terminal return approximately $50 \%$ lower than the other two groups.

Lastly, two figures are shown. Figure 6 exhibits the estimated kernel density of the LIBROCVaR (GM) strategy. The distribution is approximately symmetric. Even though it has some tails, there is a slightly heavier tail on the positive side which can be interpreted as the chance of gaining extraordinary high returns. It is not surprising that this strategy exhibits such a desirable density as it is constructed in a way to minimize heavy tails on the negative side of returns. The former results and this figure demonstrate how well that approach works.

Before, it was often mentioned that the Sharpe Ratio strategies were removed from the computations and diagrams. Figure 7 demonstrates the outlyingness for the arithmetic mean estimator, even though all three of them have a similar pattern. The exhibit shows the cumulative performance of the portfolio. DeMiguel et al. (2009) already pointed out that in empirical research it is known that the MPT strategies tend to estimate extreme weights and thus take extreme values. However, within this research only the Sharpe Ratio approaches did so. The data of this thesis contains some outliers. Nonetheless, the other strategies worked well with the data and it does not seem reasonable why specific assets, for example corn, were assigned with extreme weights in this strategy. Cumulative performances of 60 , which is equal to $6000 \%$, as well as -20 , which is equal to $-2000 \%$, are uncommon and unrealistic. Weights in this set contain values such as $42000 \%$ and $-17000 \%$. Thus, the investor would have extreme leverage and extreme, levered shortselling on specific assets. 


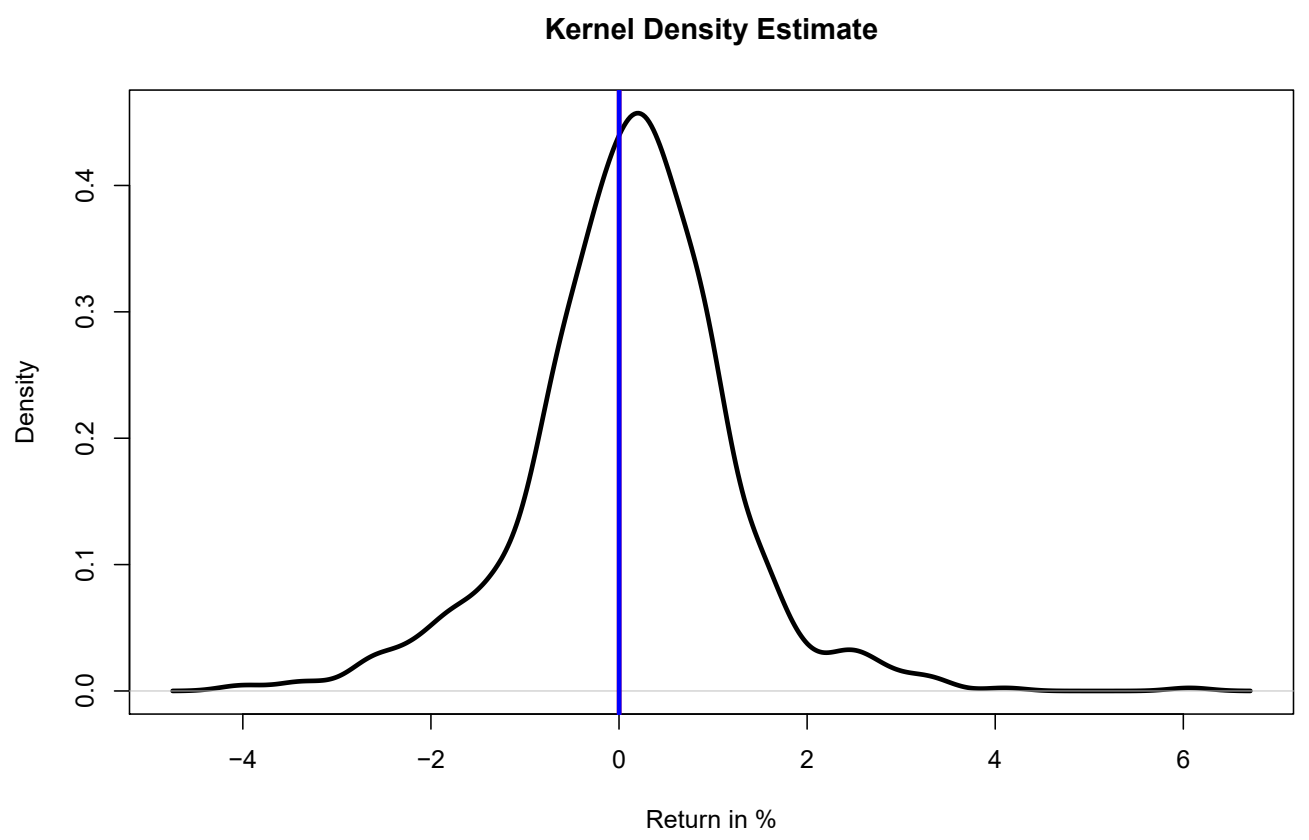

Figure 6: (Gaussian) Kernel Density Estimate of LIBRO-CVaR (GM), Median: 0.001510116, Mean: 0.00107479 Q EMAIS_Visual

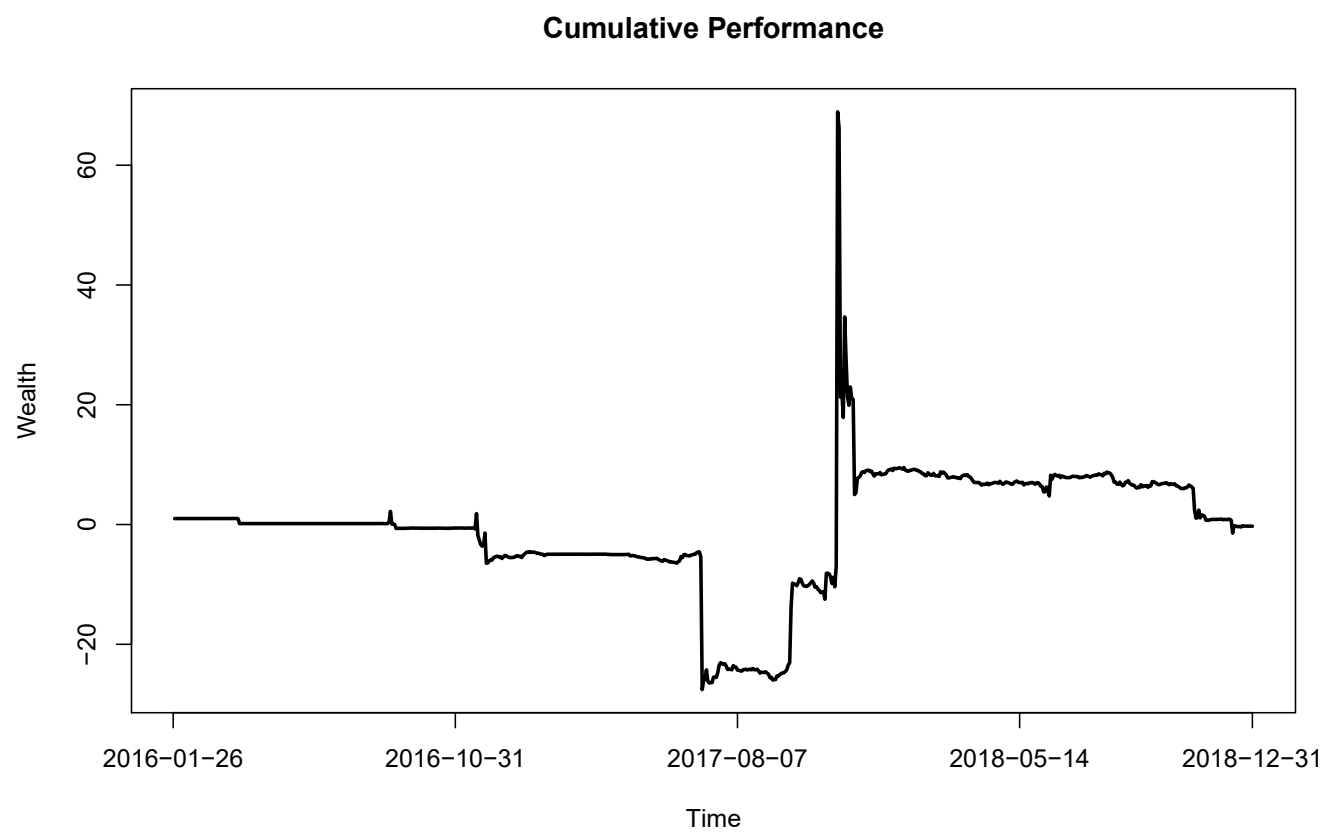

Figure 7: Cumulative Performance of Tangency Portfolio a EMAIS_Visual 


\section{Conclusion}

The process of asset allocation and portfolio analysis can usually be divided into three parts. The first one is the parameter and input estimation, the second one the allocation of wealth and the third one risk management. The focus of this thesis lies on the allocation and optimization, however the other two aspects were touched as well. Here, a brief conclusion of the methodology and empirical results is discussed.

In the methodological part different asset allocation strategies were discussed. The advantages of the CVaR strategies were described. These are the inclusion of higher moments and the actual distribution, see Petukhina et al. (2018). The empirical results demonstrated that in combination with LIBRO these portfolios come out on top of all others, supporting the usage of the actual distribution and setting a boundary for less liquid assets. The only metric where other strategies outperformed the LIBRO-CVaR was the turnover, where the maximization of the geometric mean took advantage besides the Naive Portfolio. However, these strategies do not outperform in terms of the other metrics and their performances are similar to the Naive Portfolio. This is surprising, as the approach tries to maximize terminal wealth and considers many future periods instead of only one. It can be concluded that LIBRO-CVaR and CVaR seem to be the very best strategies, but in reality an investor has to calculate whether they are efficient when trading costs are known. The MPT strategies do not perform well, which is already commonly known, see DeMiguel et al. (2009). As Fabozzi et al. (2002) state, the MPT is a normative theory, therefore the bad sample performance might come from the fact that estimators are used. On the contrary, the CVaR and LIBRO-CVaR deal well with this lack of information and do not rely on such assumptions about distribution and moments.

Furthermore, the performance of the portfolios with respect to their estimators was analyzed. Different metrics and diagrams were used, but none of the three could take a significant advantage over the other two. The geometric mean has the stablest performance among different measurements (mean, median, geometric mean). The Bayes-Stein portfolios seem to have the worst performance. Nonetheless, a clear relationship between performance and estimator was not seen.

Beyond that, one could consider an even broader investment universe to study portfolio optimization. These are, among others, international stocks, bonds as well as several alternative assets such as real estate, art, wine, musical instruments or toys. Especially the latter one is very 
interesting, as a recent study showed that Lego has, at least partially, very nice properties for a portfolio. These properties are good returns compared to stock markets and low correlation to it at the same time, see Dobrynskaya and Kishilova (2018). However, for all of the above mentioned, the limitation was lacking liquidity and lacking data which are the reasons that they are not included in this paper. Furthermore, financial instruments such as options, warrants and futures could be considered too.

This thesis extends the current state of research in the way that several methods of asset allocation are reviewed in the context of new assets such as cryptocurrencies and using different parameter estimations, as suggested by Petukhina et al. (2018). Thus, this thesis creates a basis for a more detailed analysis of the impact different asset classes have. That is, comparing how portfolios perform against each other when one asset class is left out of optimization. For example, how would the portfolios perform with and without cryptocurrencies. Furthermore, more detailed studies of parameter estimation should be conducted in order to improve portfolio performance. For example, Jacquier et al. (2003) suggest a weighted average between the geometric and arithmetic mean. Also, robust parameters such as median and median-based dispersion parameters could be considered instead of the first two moments. 


\section{References}

Artzner, P. (1999). Application of Coherent Risk Measures to Capital Requirements in Insurance. North American Actuarial Journal 3, pp. 11 -25.

Baur, D. G. and McDermott, T. K. (2010). Is gold a safe haven? International evidence. Journal of Banking \& Finance 34, pp. $1886-1898$.

Bessler, W. and Wolff, D. (2015). Do commodities add value in multi-asset portfolios? An out-ofsample analysis for different investment strategies. Journmal of Banking \& Finance 60, pp. 1 -20 .

Bessler, W., Opfer, H., and Wolff, D. (2017). Multi-asset portfolio optimization and out-of-sample performance: an evaluation of Black-Litterman, mean-variance, and naive diversification approaches. The European Journal of Finance 23, pp. 1 -30.

Bosch, D. (2017). The impact of market participants' interaction on futures prices: comparing three US wheat futures markets. International Journal of Financial Markets and Derivatives 6, pp. $120-148$.

Bulmer, M. G. (1979). Principles of Statistics.

Byrd, R., Lu, P., Nocedal, J., and Zhu, C. (1995). A Limited Memory Algorithm for Bound Constrained Optimization. SIAM Journal on Scientific Computing 16, pp. $1190-1208$.

Clarke, R., Silva, H. D., and Thorley, S. (2013). Risk Parity, Maximum Diversification, and Minimum Variance: An Analytic Perspective. The Journal of Portfolio Management 39.

DeMiguel, V., Garlappi, L., and Uppal, R. (2009). Optimal Versus Naive Diversification: How Inefficient is the 1/N Portfolio Strategy. The Review of Financial Studies 22, pp. $1915-1953$.

Dobrynskaya, V. and Kishilova, J. (2018). Lego - The Toy of Smart Investors. Available at SSRN: https: //ssrn. com/abstract=3291456.

Elton, E. J., Gruber, M. J., Brown, S. J., and Goetzmann, W. N. (2003). Modern Portfolio Theory and Investment Analysis.

Estrada, J. (2010). Geometric Mean Maximization: An Overlooked Portfolio Approach? The Journal of Investing 19, pp. $134-147$.

Fabozzi, F. J., Gupta, F., and Markowitz, H. M. (2002). The Legacy of Modern Portfolio Theory. The Journal of Investing 11.

Glaser, F., Zimmermann, K., Haferkorn, M., Weber, M. C., and Siering, M. (2014). Bitcoin - Asset or Currency? Revealing Users' Hidden Intentions. Available at SSRN: https://ssrn.com/abstract=2425247.

Goldfarb, D. and Idnani, A. (1983). A Numerically Stable Dual Method for Solving Strictly Convex Quadratic Programs. Mathematical Programming 5, pp. 1 -33. 
Jacquier, E., Kane, A., and Marcus, A. J. (2003). Geometric or Arithmetic Mean: A Reconsideration. Financial Analysts Journal 59, pp. $46-53$.

James, W. and Stein, C. (1961). Estimation with Quadratic Loss. 4th Berkeley Symposium on Probability and Statistics 1, pp. $361-379$.

Jiao, W. (2003). Portfolio Resampling and Efficiency Issues. MA thesis. Humboldt-Universität zu Berlin, Wirtschaftswissenschaftliche Fakultät. DOI: http://dx.doi.org/10.18452/14039.

Jorion, P. (1986). Bayes-Stein Estimation for Portfolio Analysis. Journal of Financial and Quantitative Analysis 21, pp. $279-292$.

Klein, T., Thu, H. P., and Walther, T. (2018). Bitcoin is not the New Gold - A comparison of volatility, correlation, and portfolio performance. International Review of Financial Analysis 59, pp. $105-116$.

Krokhmal, P., Palmquist, J., and Uryasev, S. (2003). Portfolio Optimization With Conditional Value-at-Risk Objective And Constraints. Journal of Risk 4, pp. 43 -68.

Lai, T. L., Xing, H., and Chen, Z. (2011). Mean-Variance Portfolio Optimization When Means and Covariances are Unknown. The Annals of Applied Statistics 5, pp. 798 -823.

Markowitz, H. (1952). Portfolio Selection. The Journal of Finance 7, pp. 77 -91.

Nelder, J. and Mead, R. (1965). A Simplex Algorithm for Function Minimization. The Computer Journal 7, pp. $308-313$.

Petukhina, A., Trimborn, S., Härdle, W. K., and Elendner, H. (2018). Investing with cryptocurrencies - evaluating the potential of portfolio allocation. Available at SSRN: https://ssrn. com/abstract $=3274193$.

Pézier, J. and White, A. (2008). The relative merits of investable hedge fund indices and of funds of hedge funds in optimal passive portfolios. The Journal of Alternative Investments 10, pp. 37 -49 .

Razali, N. M. and Yap, B. W. (2011). Power Comparisons of Shapiro-Wilk, Kolmogorov-Smirnov, Lilliefors and Anderson-Darling Tests. Journal of Statistical Modeling and Analytics 2, pp. 21 -33 .

Rockafellar, R. T. and Uryasev, S. (2000). Optimization of Conditional Value-at-Risk. Journal of Risk 2, pp. $21-41$.

Scott, F. and Yelowitz, A. (2010). Pricing Anomalies in the Market for Diamonds: Evidence of Conformist Behavior. Economic Inquiry 48, pp. 353 -368.

Sharpe, W. F. (1966). Mutual Fund Performance. The Journal of Business 39, pp. $119-138$.

Stein, C. (1955). Inadmissibility of the Usual Estimator for the Mean of Multivariate Normal Distribution. 3rd Berkeley Symposium on Probability and Statistics 1, pp. 197 -206. 
Stonehouse, J. M. and Forrester, G. J. (1998). Robustness of the $\mathrm{t}$ and $\mathrm{U}$ tests under combined assumption violations. Journal of Applied Statistics 25, pp. $63-74$.

Toutenburg, H. and Heumann, C. (2008). Induktive Statistik - Eine Einführung mit R und SPSS.

Trimborn, S., Li, M., and Härdle, W. K. (2019). Investing with Cryptocurrencies - A Liquidity Constrained Investment Approach. Journal of Financial Econometrics, pp. 1 -27. 


\section{Appendix A - Asset Classes}

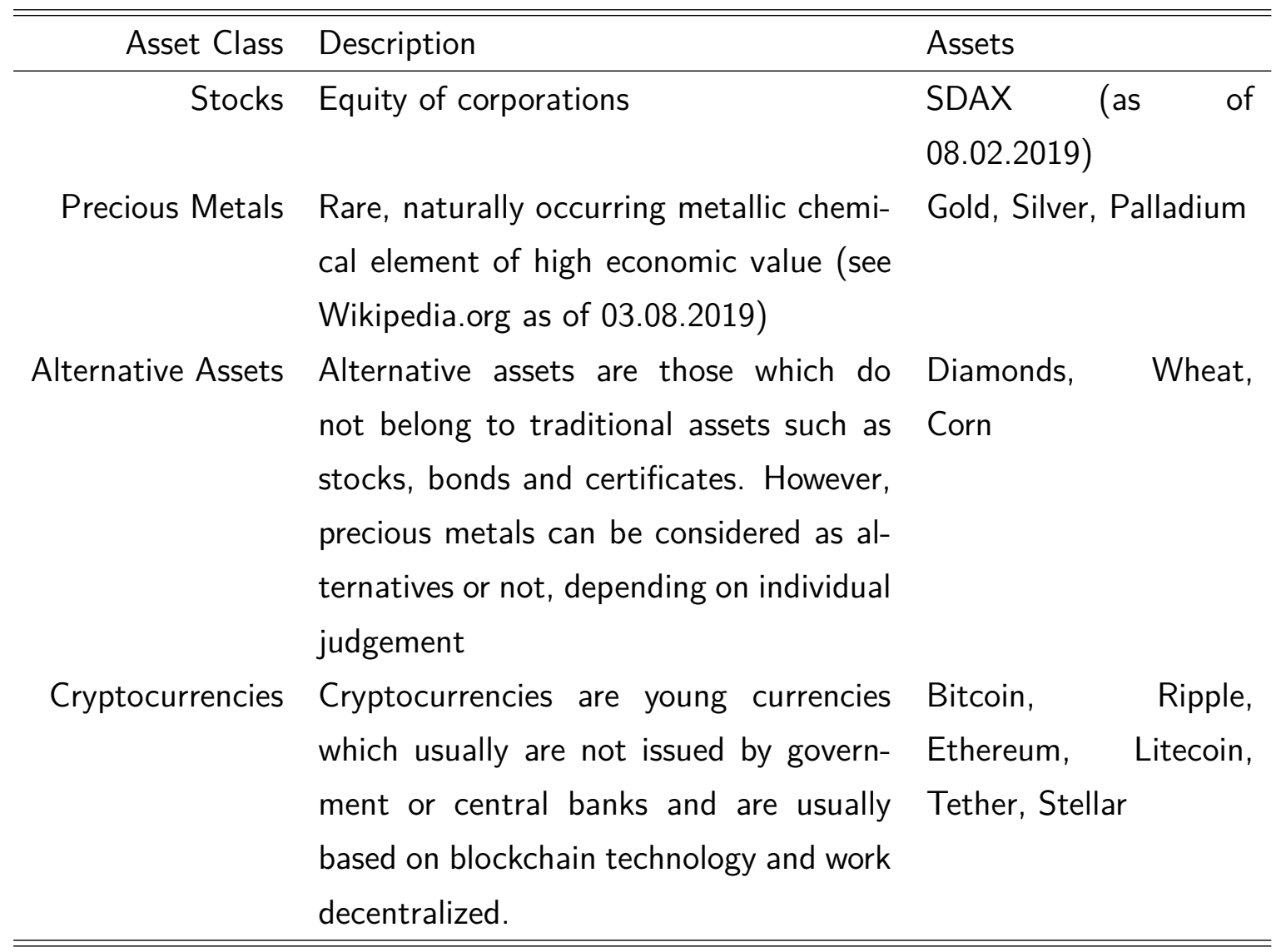

Table 3: Description of Asset Classes used 


\section{Appendix B - Descriptive Statistics}

\begin{tabular}{|c|c|c|c|c|c|c|c|c|c|c|}
\hline & Min & $\begin{array}{l}\text { 1st Quar- } \\
\text { tile }\end{array}$ & Median & $\begin{array}{l}\text { Arithmetic } \\
\text { Mean }\end{array}$ & $\begin{array}{l}\text { Geometric } \\
\text { Mean }\end{array}$ & $\begin{array}{l}\text { 3rd Quar- } \\
\text { tile }\end{array}$ & Max & $\begin{array}{l}\text { Standard } \\
\text { Deviation }\end{array}$ & Skewness & Kurtosis \\
\hline 1 & -0.0397 & -0.0038 & 0.0013 & 0.0008 & 0.0040 & 0.0059 & 0.0561 & 0.0090 & -0.1297 & 5.8391 \\
\hline 2 & -0.0533 & -0.0017 & 0.0000 & 0.0000 & 0.0016 & 0.0020 & 0.0314 & 0.0056 & -0.8489 & 16.6920 \\
\hline 3 & -0.0498 & -0.0018 & 0.0000 & 0.0000 & 0.0007 & 0.0022 & 0.0315 & 0.0055 & -0.6738 & 15.0582 \\
\hline 4 & -34.3472 & -0.0113 & -0.0001 & -0.0586 & 0.0046 & 0.0095 & 4.4919 & 1.3469 & -22.2189 & 556.4377 \\
\hline 5 & -0.0395 & -0.0037 & 0.0013 & 0.0008 & 0.0042 & 0.0059 & 0.0593 & 0.0091 & -0.0649 & 6.1692 \\
\hline 6 & -0.0401 & -0.0047 & 0.0010 & 0.0007 & 0.0051 & 0.0065 & 0.0526 & 0.0101 & -0.1124 & 5.7179 \\
\hline 7 & -0.0498 & -0.0019 & 0.0000 & 0.0001 & 0.0014 & 0.0021 & 0.0315 & 0.0056 & -0.6236 & 14.4252 \\
\hline 8 & -0.0449 & -0.0044 & 0.0014 & 0.0011 & 0.0051 & 0.0074 & 0.0742 & 0.0108 & 0.0730 & 6.7591 \\
\hline 9 & -0.0377 & -0.0047 & 0.0013 & 0.0009 & 0.0050 & 0.0071 & 0.0747 & 0.0107 & 0.1583 & 6.5797 \\
\hline 10 & -0.0533 & -0.0018 & 0.0000 & 0.0000 & 0.0016 & 0.0021 & 0.0315 & 0.0056 & -0.8484 & 16.7995 \\
\hline 11 & -0.0498 & -0.0018 & 0.0000 & 0.0000 & 0.0007 & 0.0022 & 0.0315 & 0.0055 & -0.6738 & 15.0582 \\
\hline 12 & -24.2532 & -0.0124 & 0.0000 & -0.0238 & 0.0050 & 0.0100 & 5.9647 & 0.9134 & -24.0747 & 651.5108 \\
\hline 13 & -0.0398 & -0.0037 & 0.0013 & 0.0008 & 0.0041 & 0.0059 & 0.0588 & 0.0091 & -0.0776 & 6.1277 \\
\hline 14 & -0.0389 & -0.0044 & 0.0008 & 0.0006 & 0.0000 & 0.0060 & 0.0364 & 0.0092 & -0.2980 & 5.0623 \\
\hline 15 & -0.0498 & -0.0019 & 0.0000 & 0.0001 & 0.0014 & 0.0021 & 0.0315 & 0.0056 & -0.6225 & 14.4771 \\
\hline 16 & -0.0410 & -0.0048 & 0.0015 & 0.0011 & 0.0052 & 0.0073 & 0.0607 & 0.0108 & -0.0218 & 5.3774 \\
\hline 17 & -0.0425 & -0.0046 & 0.0013 & 0.0010 & 0.0050 & 0.0072 & 0.0949 & 0.0109 & 0.5913 & 10.9381 \\
\hline 18 & -0.0556 & -0.0021 & 0.0000 & 0.0000 & 0.0021 & 0.0025 & 0.0314 & 0.0061 & -0.7320 & 14.2314 \\
\hline 19 & -0.0498 & -0.0018 & 0.0000 & 0.0000 & 0.0007 & 0.0022 & 0.0315 & 0.0055 & -0.6738 & 15.0582 \\
\hline 20 & -12.7098 & -0.0060 & 0.0000 & -0.0218 & 0.0024 & 0.0046 & 2.3579 & 0.5112 & -20.8450 & 507.3358 \\
\hline 21 & -0.0395 & -0.0038 & 0.0013 & 0.0008 & 0.0041 & 0.0059 & 0.0592 & 0.0091 & -0.0653 & 6.1563 \\
\hline 22 & -0.0492 & -0.0034 & 0.0002 & 0.0002 & 0.0000 & 0.0047 & 0.0534 & 0.0086 & -0.3856 & 9.4273 \\
\hline 23 & -0.0498 & -0.0021 & 0.0000 & 0.0001 & 0.0015 & 0.0025 & 0.0315 & 0.0060 & -0.4591 & 12.1127 \\
\hline 24 & -0.0481 & -0.0044 & 0.0015 & 0.0011 & 0.0052 & 0.0071 & 0.1023 & 0.0110 & 0.7409 & 13.0017 \\
\hline 25 & -0.0446 & -0.0046 & 0.0013 & 0.0010 & 0.0050 & 0.0076 & 0.0668 & 0.0108 & -0.0038 & 5.6813 \\
\hline
\end{tabular}

Table 4: Descriptive Statistics for all Strategies a EMAIS_Visual 


\begin{aligned} \hline \hline Number & Strategy \\ \hline 1 & Naive Portfolio \\ 2 & Constrained Minimum Variance (AM) \\ 3 & Global Minimum Variance (AM) \\ 4 & Sharpe Ratio (AM) \\ 5 & F-Geometric Mean Maximization (AM) \\ 7 & C-Geometric Mean Maximization (AM) \\ 8 & LIBRO-CVaR (AM) \\ 9 & CVaR (AM) \\ 10 & Constrained Minimum Variance (GM) \\ 11 & Global Minimum Variance (GM) \\ 12 & Sharpe Ratio (GM) \\ 13 & F-Geometric Mean Maximization (GM) \\ 14 & C-Geometric Mean Maximization (GM) \\ 15 & LIBRO-MPT (GM) \\ 16 & LIBRO-CVaR (GM) \\ 17 & CVaR (GM) \\ 18 & Constrained Minimum Variance (BS) \\ 19 & Global Minimum Variance (BS) \\ 20 & Sharpe Ratio (BS) \\ 21 & F-Geometric Mean Maximization (BS) \\ 22 & C-Geometric Mean Maximization (BS) \\ 23 & LIBRO-MPT (BS) \\ 24 & LIBRO-CVaR (BS) \\ \hline \hline & CVaR (BS) \\ \hline & \end{aligned}

Table 5: Numbers and Names of Strategies for table 4 a. EMAIS_Visual 


\section{Declaration of Honesty}

I, Erin Dirk Sprünken, hereby declare that I have not previously submitted the present work for other examinations. I wrote this work independently. All sources, including sources from the Internet, that I have reproduced in either an unaltered or modified form (particularly sources for texts, graphs, tables and images), have been acknowledged by me as such. I understand that violations of these principles will result in proceedings regarding deception or attempted deception.

Hiermit erkläre ich, Erin Dirk Sprünken, dass ich die vorliegende Arbeit noch nicht für andere Prüfungen eingereicht habe. Ich habe die Arbeit selbständig verfasst. Sämtliche Quellen einschließlich Internetquellen, die ich unverändert oder abgewandelt wiedergegeben habe, insbesondere Quellen für Texte, Grafiken, Tabellen und Bilder, habe ich als solche kenntlich gemacht. Ich bin mir darüber bewusst, dass bei Verstößen gegen diese Grundsätze ein Verfahren wegen Täuschungsversuchs bzw. Täuschung eingeleitet wird.

Signature

Erin Dirk Sprünken

Berlin, September 8, 2019 